\title{
Tuning muscarinic receptor signalling
}

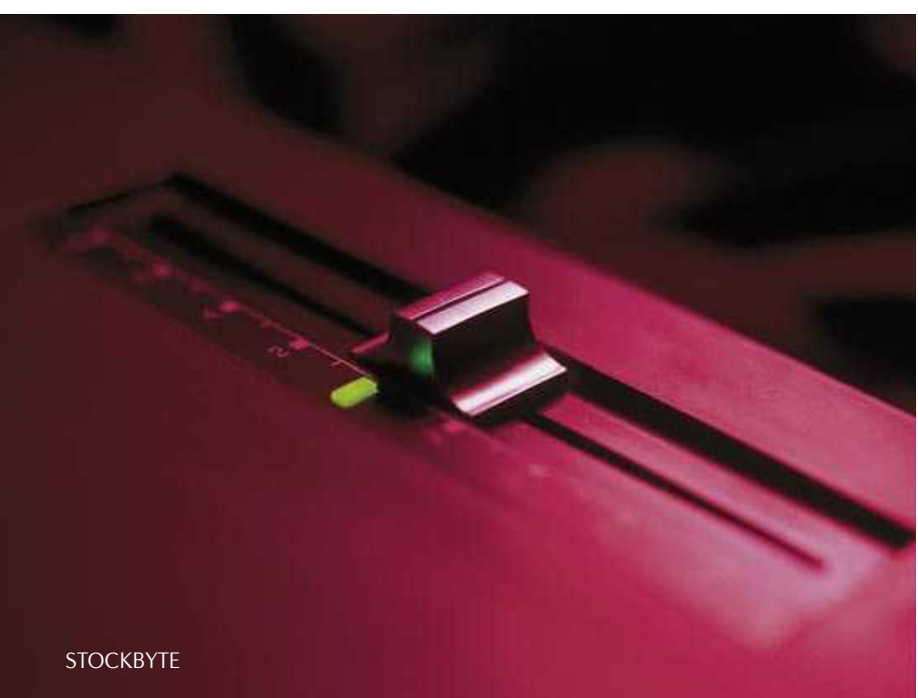

The pharmacotherapy of schizophrenia is currently dominated by atypical antipsychotics that primarily target dopaminergic signalling, the dysregulation of which has long been considered to have a key role in the disease. However, although these agents can ameliorate positive disease symptoms such as hallucinations, they have little efficacy against other aspects such as cognitive deficits. Moreover, they have also been associated with poor tolerability and side effects such as weight gain.

More recently, a muscarinic hypothesis of schizophrenia has emerged, as experiments with muscarinic acetylcholine receptor knockout mice have implicated the $M_{1}$ and $M_{4}$ receptor subtypes in the modulation of both dopamine and glutamate transmission in areas of the brain that are involved in psychosis. However, the lack of specific pharmacological modulators of muscarinic receptors has hampered validation of this hypothesis. Now, in PNAS, Felder and colleagues report the discovery of a smallmolecule modulator - LY2033298 - that is specific for the $M_{4}$ receptor subtype, which shows promising effects in preclinical models of schizophrenia.

Previous attempts to selectively modulate muscarinic receptors by targeting the acetylcholine binding site have been largely unsuccessful, mainly because this site is highly conserved across the different receptor subtypes. Here, the authors show that targeting the more divergent allosteric binding sites, which are topographically distinct from the acetylcholine binding site, allows selective modulation of a particular muscarinic receptor subtype to be achieved.

Using an assay that is based on monitoring intracellular calcium signalling in cell lines expressing a particular muscarinic receptor subtype, the authors found that LY2033298 enhanced acetylcholine responses specifically at the $\mathrm{M}_{4}$ subtype. Radioligand binding assays showed that LY2033298 potentiated acetylcholine binding, but did not affect antagonist binding. Also, functional screening of $\mathrm{M}_{4}$ receptor mutants revealed that Asp432 is a crucial residue for LY2033298mediated potentiation.

Finally, the authors showed that LY2033298 had activity in rodent models predictive of antipsychotic efficacy. It attenuated conditioned avoidance responding in trained rats and reversed the apomorphineinduced suppression of the acoustic startle response. These results indicate that positive allosteric modulation of the $\mathrm{M}_{4}$ muscarinic receptor could be a promising new strategy for treating schizophrenia, and that compounds such as LY2033298 might represent a novel class of antipsychotic drugs.

Monica Hoyos Flight

ORIGINAL RESEARCH PAPER Chan, W. Y. et al.

Allosteric modulation of the muscarinic

$\mathrm{M}_{4}$ receptor as an approach to treating schizophrenia. Proc. Natl Acad. Sci. USA 105, 10978-10983 (2008)

FURTHER READING Wess, J., Eglen, R. M. $\varepsilon$ Gautam, D. Muscarinic acetylcholine receptors: mutant mice provide new insights for drug development. Nature Rev. Drug Discov. 6, 721-733 (2007) 